\title{
Design for Environmental Three-Dimensional Parking Equipment
}

\author{
Min Zhang ${ }^{a}$, Songlin Zhang ${ }^{b^{*}}$, Jia Jin ${ }^{c}$ and Pengpeng Wang ${ }^{d}$ \\ College of Geography and Environmental Science, Northwest Normal University, Lanzhou, P. R. \\ China, 730070 \\ a344055830@qq.com, bzhangsonling65@163.com, '83634283@qq.com, 'ztawpp@163.com \\ *The corresponding author
}

Keywords: Easy to assemble; Disassembled; Flexible; Portable; Environmental

\begin{abstract}
In this paper, a kind of environmental three-dimensional parking equipment was designed, which was flexible, easy to assemble, disassembled and portable. As a result, the vertical and transverse movements of the parked vehicles were realized in need. Easy assembled and removable steel structure was used to make the main body of the equipment, and the steel structure was also reconstructed in any apparent form according to the conditions of the resources from the land and the space in the construction site; The whole device could be moved because the related artifacts were not only reused but also disassembled and assembled for many times, which made the time, human resource and material cost be saved. At last, extremely precious resources of the land and the space in the city were saved, the use ratio of which was also improved. In addition, the traffical congestion was reduced, and the emissions of pollutants like the emission of vehicle exhaust were done as well.
\end{abstract}

\section{Introduction}

With the rapid development of economy and society, the resources of the urban land and space were becoming scarcer and more expensive than before. At the same time, the rising amount of car ownership and road trips [1] leads to the high demand for parking Spaces and the apparent phenomenon that there was no place to park. It's very common about the temporary and long-term parking on the "No Parking" area, affecting the utilization of the resources from the land and the space in other forms besides the city traffic [2].

The limited resources of the land and the space in the city should be fully used to design and develop the environmental three-dimensional parking facility, which was deformable, easy to assemble, removable and portable; And the facility was also suitable for various forms of the land and space resources. The limited resources of the land and the space refer to the edge space from the municipal, industrial and commercial and residential environments, with the small area, irregular and scattered distribution, as well as those from some large and medium-sized stereo parking equipment which were hard to built. Using the stereo parking facility, the parking problem of our country would be solved [3]. To sum up, the structure of the parking equipment, with the characteristics above and the realizing forms of parts of the functions, were designed by ourselves.

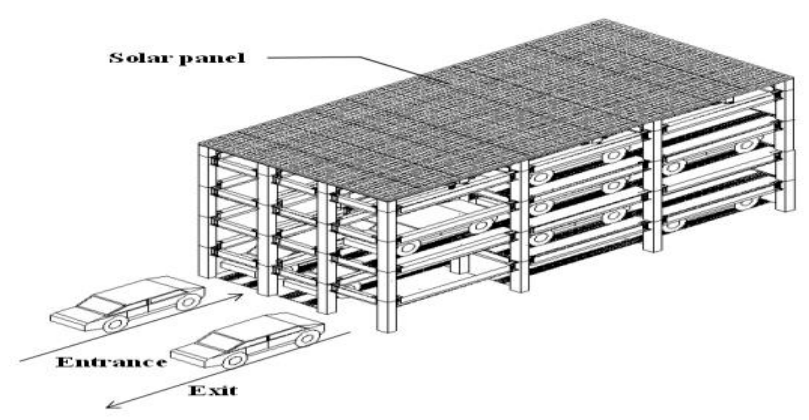

Figure 1. Main structure of assembled and disassembled three-dimensional parking facility 


\section{Main Structure of Assembled and Disassembled Three-dimensional Parking Facility}

This parking equipment was made up of 4 parts, such as the easy-assembled, removable and deformable steel structure, the vertical and transverse movement structure, the control system and the security device. In recent years, new energy vehicles gradually popularized, solar panel was added to the top of the three-dimensional parking equipment, to provide electricity for not only the electric cars but the control system of the stereo parking equipment, like the motor. As is shown in Fig. 1 .

Design for the Detachable and Attachable Steel Structure. As a bearing structure, main steel framework included the H-beam and the stand column welded with the $140 \mathrm{~mm}$ hollow square steel or connected with the bolt of the friction-type and high-strength. Meanwhile, stiffener plates were added to the beam and stand column in order to effectively increase their strength and stiffness. The stand column was fixed on the foundation through the anchor bolts to ensure the whole security and stability of the parking equipment $[4,5]$.

The three-dimensional parking equipment was made up of multiple parking units, and it would be assembled according to the size and shape of the construction site. The type of the parked vehicle was determined by the size and shape of the parking unit. According to the building and designing specifications of the parking equipment (JGJ100-2015), the length $\mathrm{x}$ width $\mathrm{x}$ height of the parking unit was designed. A parking unit was a parking space, including stand column, longitudinal girder, transverse girder and carry-board. As is shown in Fig. 2 .
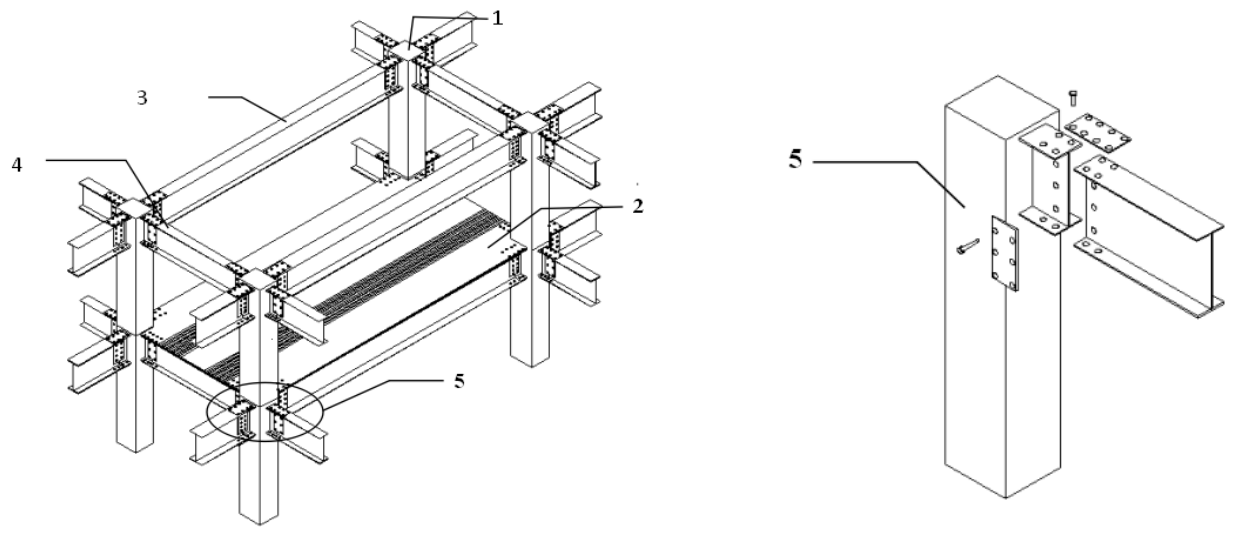

1-Stand column; 2-Carry-board; 3-Transverse girder; 4-Longitudinal girder; 5Detachable connection point

Figure 2. The diagram of parking unit

The Structure of Vertical and Transverse Movement of Three-Dimensional Parking Equipment. The structure of vertical and transverse movement of the three-dimensional parking equipment consisted of two parts, vertical and transverse moving devices. Relying on the mobile platform, the vehicle was transferred respectively along the $\mathrm{X}$-axis, Y-axis or Z-axis in the parking equipment [6]. As is shown in Fig. 3 .

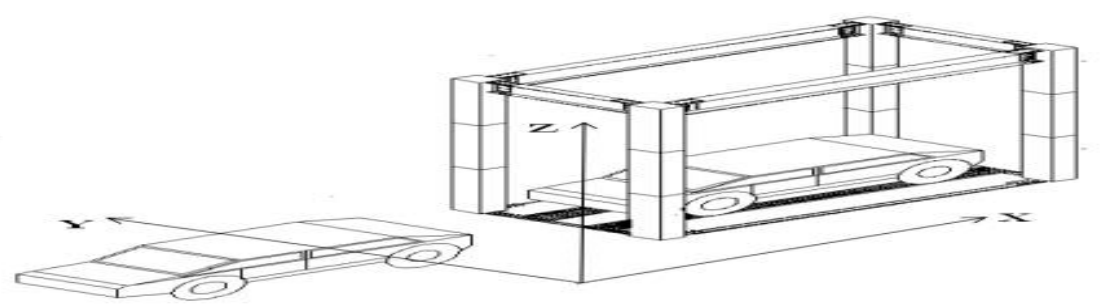

Figure 3. The movement along the axes 
There were the light rail, mobile sprocket and mobile guiding roller mainly in X-axis; The motor, reducer, transverse moving rail, transverse moving sprocket, driving gear and rack were mainly included in Y-axis; To ensure the stability of the transport along the $\mathrm{X}$-axis and $\mathrm{Y}$-axis, the carry-board was moved by the transverse driving motor leading the devices of the $\mathrm{X}$-axis and the $\mathrm{Y}$-axis through the movements of the transverse moving sprocket and chains along the axes. As is shown in Fig. 4 and Fig. 5 .

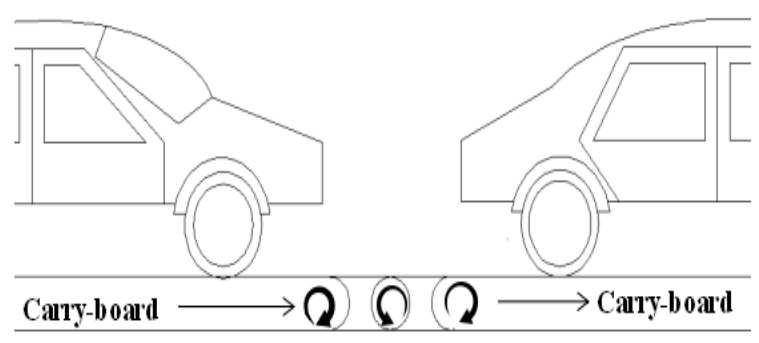

Figure 4. Device of X-axis

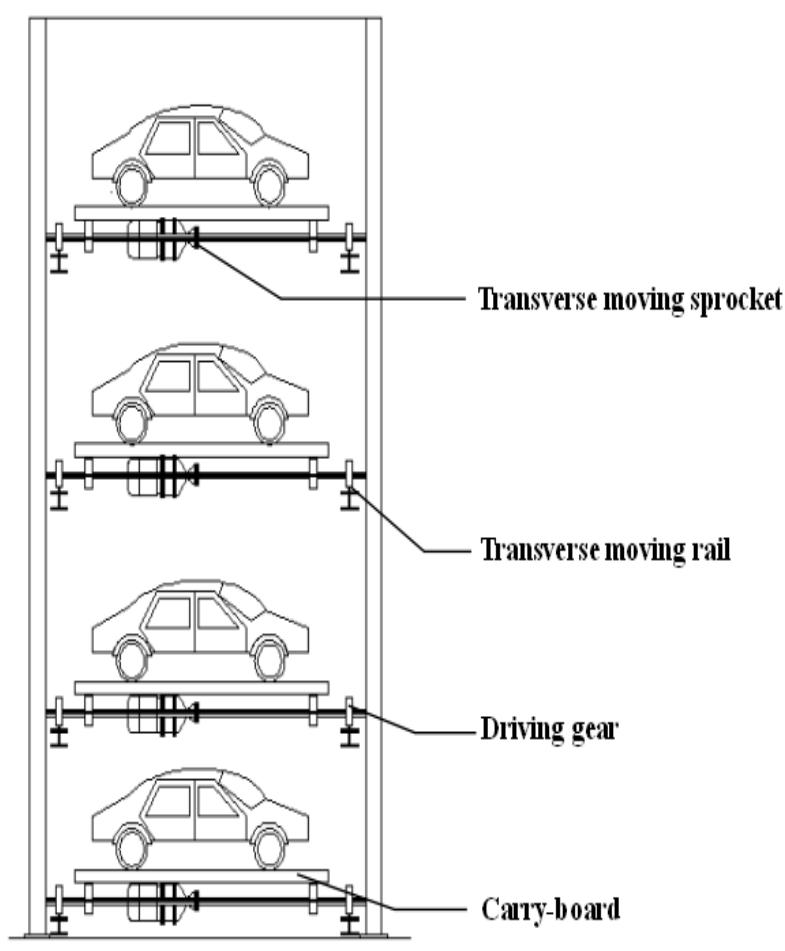

Figure 5. Device of Y-axis

The motor, guardrail, reducer, transmission system and parallel guide rail were mainly included in the Z-axis, the movement along the Z-axis was performed by the driving wheel and the steel rope; The motor driven by the driving wheel, the steel rope convolved on the roller was used to complete the vertical movement of the carry-board, in order to ensure the work along the Z-axis [7]. As is shown in Fig. 6 . 


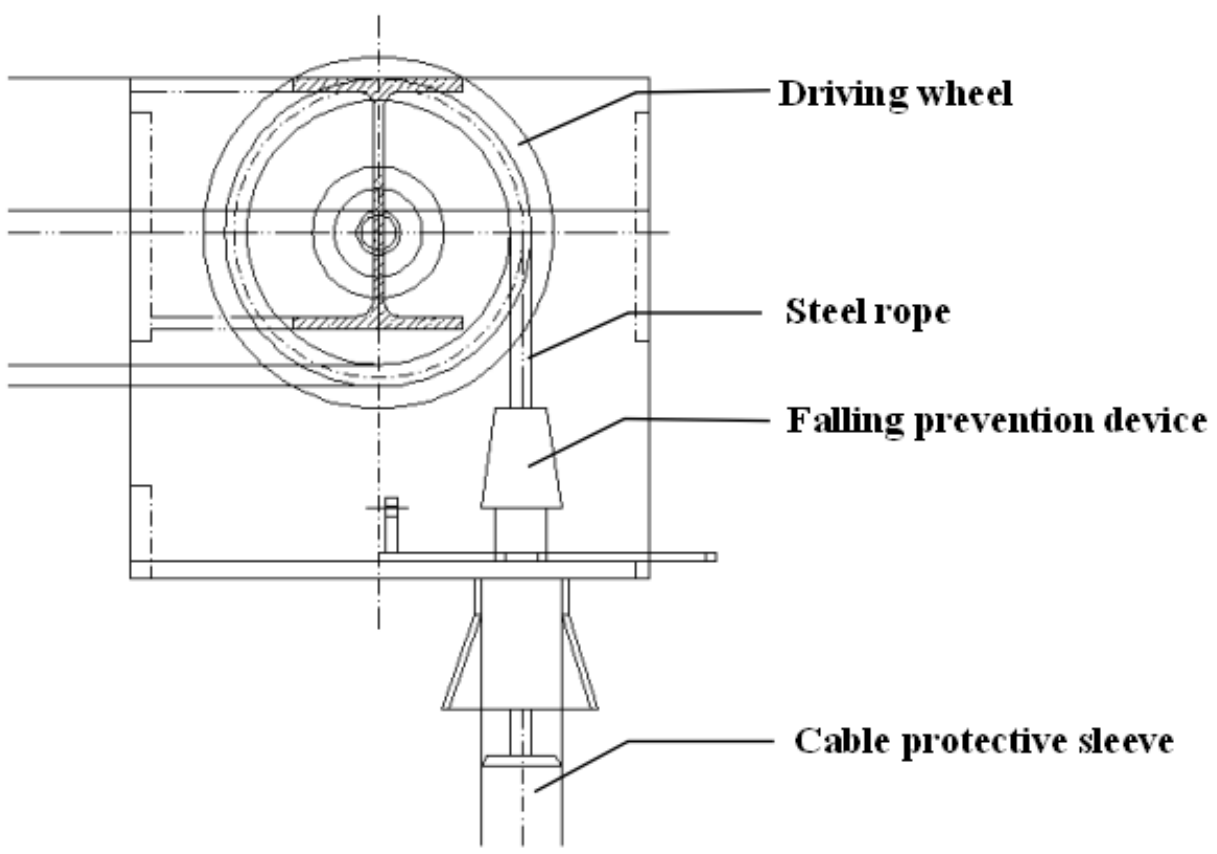

Figure 6. Device of Z-axis

Control System of Three-Dimensional Parking Equipment. The automatic control system PLC was adopted to detect the running state of the parking space and other parameters in the parking equipment, so as to control the motor to work reasonably in turn, making the running smooth and safety, the operation simple and convenient [8].

Safety Protection Measures. During the operation of the stereo parking equipment, the corresponding protection measures were taken because there maybe a variety of security issues. First of all, the location, length, width, height, and weight of the vehicle should be detected automatically on the mobile platform, avoiding the entering of the overstepping vehicle, or else the system would automatically alert [9]. Then, the reducer and falling prevention device should be installed, to prevent speeding and falling during the process of the transverse and lifting movement. In the end, as the last security device of the parking equipment, the buffer at the bottom of the carrier-board was used to deal with the running issues of the equipment, such as the chain fracture, the lack of power and the trouble of the control system.

\section{Basic Working Principle of Three-Dimensional Parking Equipment}

Parked on the carrier-board, the front and rear wheels of the vehicle were respectively adjusted and pushed by the adjuster of the mobile platform, after adjustment the vehicle was moved to the specified location. When the mobile platform went up to the specified floor, guided by the transverse device of the mobile platform, move the vehicle from the carrier-board into the parking unit. While the car were fetched, it was ca back to the mobile platform by the front and rear guiding rollers [10]. The processes of parking and fetching were completed by the automatic control system PLC. As is shown in both Fig. 7 and Fig. 8 . 


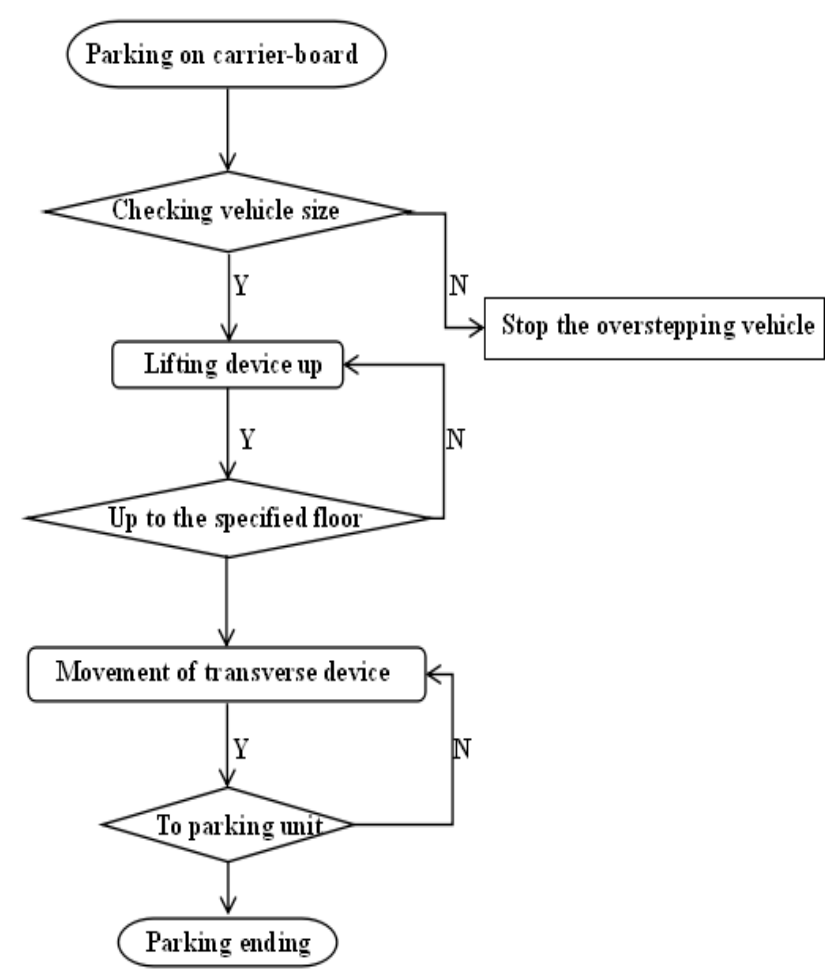

Figure 7. Flow chart of parking

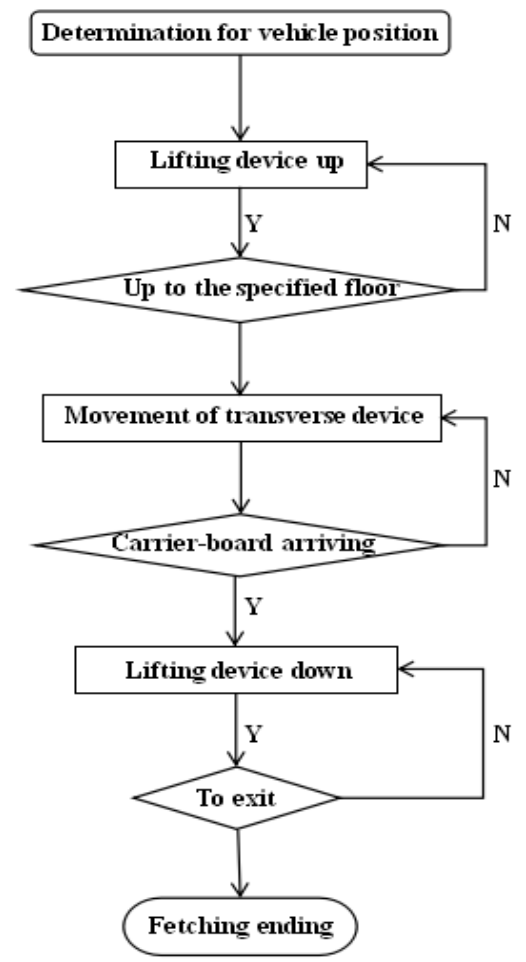

Figure 8. Flow chart of fetching

\section{Conclusion}

In this paper, a kind of environmental three-dimensional parking equipment was designed, which was flexible, easy to assemble, removable and portable. Using the stereo parking facility, the urban parking and fetching problems were effectively solved. Through the sufficient and reasonable use of the urban land and space resources, the land, space, and human resources were greatly saved. The parking space would be increased or reduced according the increasing and reducing of the parked vehicles. This parking equipment, with the simple structure, was conveniently disassembled and assembled. The vertical and transverse movements of the vehicle were completed by the steel rope and the driving wheel, with the low cost. When the mobile platform was in no-load, the lifting and dropping speeds were improved through the control system, by which the parking time was also reduced and the parking efficiency was improved. The lock catch and the falling prevention device were installed between the mobile platform and the steel rope, avoiding the slipping and falling of the vehicle.

\section{Acknowledgements}

The authors are very grateful for the financial support from the National Natural Science Fund of China (51068025), the Natural Science Fund of Gansu Province in China (1308RJZA302), and the Open Research Fund of Oil and Gas Resources Key Laboratory of Gansu Province in China (SZDKFJJ20150606).

\section{References}

[1] Y.Y. Wang: Super Science , (2016) No.2, p.299. (In Chinese)

[2] Z.J. Liu: Research on Stereo Parking Lot Based on the Hanging PRT (MS., North China University of Technology, China, 2016)

[3] J.Wang: ChengShi Jianshe LiLun Yan Jiu, Vol. 5 (2015) No.29, p.212. (In Chinese) 
[4] B.J. Wu, Z.F. Shi: Journal of Jimei University (Natural Science), (2014) No.2, p.133. (In Chinese)

[5] Roger: Architectural Journal, (2013) No.1, p.9. (In Canada)

[6] X.Y. Liang, C.C. Duan: Manufacturing Automation, Vol.32 (2010) No.2, p.181. (In Chinese)

[7] J.Y. Su: Development \& Innovation of Machinery \& Electrical Products, Vol.28 (2015) No.1, p.17. (In Chinese)

[8] L.Tao, J.T. Wu: Hoisting and Conveying Machinery, (2015) No.1, p.14. (In Chinese)

[9] L.J. Miao, Z.W. Wang: Hoisting and Conveying Machinery, (2014) No.5, p.7. (In Chinese)

[10] J.H. Wang, Y.Y. Ma: Journal of Chongqing University of Science and Technology (Natural Science), Vol.18 (2016) No.1, p.24. (In Chinese) 